\title{
Factores influyentes en la implementación de un plan lector en el nivel medio mayor de un jardín infantil de la comuna de Lampa, vía transferencia de fondos JUNJI
}

\author{
Ailyn Bernal Puebla ${ }^{1}$ \\ Andrea Cifuentes Inzunza² \\ Andrea Montt Soto ${ }^{3}$ \\ Elizabeth Quezada Guevara ${ }^{4}$
}

Recibido 25 de febrero 2018 · Aceptado de 18 abril 2019

\begin{abstract}
RESUMEN.
El presente artículo describe la experiencia obtenida en un jardín infantil de Chile sobre los factores que influyen en la implementación de un plan lector, y cómo estos inciden, positiva o negativamente, en el proceso de ejecución para el desarrollo de las habilidades de comprensión lectora de niños y niñas. Se empleó metodología cualitativa, llevándose a cabo entrevistas y registros de observación. Los resultados permitieron observar, primeramente, que existe un desconocimiento por parte del equipo educativo del nivel respecto de la política o programa que sustenta el plan lector; además, se detectaron diversas visiones y realidades en las prácticas de los actores que participan en la institución educativa.
\end{abstract}

Palabras clave: pruebas estandarizadas, comprensión lectora, plan lector, interés por la lectura, evaluación.

Influential Factors in the Implementation of a Reading Plan in a Preschool in Lampa, by the Transfer of JUNJI Funds

\section{ABSTRACT.}

This article describes the experience of a Chilean preschool regarding the factors that influence the implementation of a reading plan, and how these positively or negatively affect the execution process for the development of children's reading comprehension skills. A qualitative methodology was applied, using interviews and observation records. First and foremost, the results revealed that there is a lack of knowledge in the educational team regarding the policy or program supporting the reading plan, and different visions and realities were detected in the practices of those participating in the educational institution.

Key words: standardized tests, reading comprehension, reading plan, interest in reading, evaluation.

Educadora de Párvulos, Casa Nacional del Niño-SENAME. Contacto: ailyn_I8@hotmail.com Educadora de Párvulos, Fundación Integra. Contacto: cifuentes.in@gmail.com

Educadora de Párvulos. Magíster en Educación, mención Didáctica del Lenguaje y las Matemáticas. Junta Nacional de Jardines Infantiles. Contacto: andreamontt@gmail.com

4 Educadora de Párvulos. Colegio Verbo Divino de Chicureo. Contacto: ely.quezada.g@gmail.com 


\section{Introducción}

La presente investigación surge de la necesidad de indagar acerca del impacto práctico que tiene la política pública de lectura del gobierno de la presidenta Michelle Bachelet, Plan Nacional de la Lectura, cuya misión es reconocer y visibilizar la lectura como un derecho que contribuye a acortar las brechas sociales y culturales dentro del país, promoviendo el acceso a diversas prácticas y soportes de la lectura a lo largo de todo el ciclo de vida y formando personas informadas, críticas, creativas, reflexivas y participativas.

El desarrollo del lenguaje es uno de los objetivos fundamentales de la educación, ya que es la principal herramienta mediante la cual el ser humano construye y comprende el mundo que lo rodea, y para alcanzar este desafío la lectura cumple sin duda un lugar protagónico. Por esto, es necesario indagar en los factores que influyen en la implementación eficaz de un plan lector para el desarrollo de habilidades lectoras en niños y niñas chilenos.

El presente escrito describe las características de los principales planes lectores exitosos, nacionales e internacionales. También muestra los factores influyentes en el desarrollo de la lectura, las estrategias de lectura desde el punto de vista de algunos autores y, finalmente, relata la experiencia obtenida en 2017 en un jardín infantil de la comuna de Lampa en Chile.

\section{Marco teórico}

\section{Situación actual de la lectura en Chile}

Por muchos años la alfabetización se concibió como un área de aprendizaje al que solo algunos podían acceder; aquellos privilegiados, por ser de un estatus socioeconómico alto, participaban en jornadas en las que se les enseñaba el sentido de los signos que componían un escrito, a lo que se denominaba "leer". Luego, al pasar el tiempo, fueron más los que pudieron acceder a este tesoro de conocimiento y fue así como personas de diversos estratos sociales y de distintas edades comenzaron a ser instruidas para poder decodificar signos 
que para ese entonces era lo necesario para ser considerado como una persona alfabetizada.

Acorde con las nuevas políticas públicas del siglo XX, se dicta la Ley de Instrucción Primaria Obligatoria para todo niño y niña entre 7 y I5 años de edad, con cuatro años de obligatoriedad, los que ascenderían a seis años de obligatoriedad en 1929. Luego, en 1965, la Reforma Educacional extiende a ocho años la escolaridad obligatoria, lo que significaría un gran avance en materia de cobertura en educación para la población chilena, siendo estas políticas importantes para el crecimiento del país.

Con mayor cantidad de participantes en las aulas, se expande la posibilidad para acceder a instancias educativas en las que se imparten las distintas materias correspondientes a las áreas más formales de la educación. En la tabla I queda en evidencia el aumento de cobertura en el periodo 1992 a 2002.

Tabla I. Porcentaje de escolaridad a nivel nacional

\begin{tabular}{|l|c|c|c|c|}
\hline AÑO & EDUCACIÓN & NUNCA ASISTIÓ & & \\
\hline 1992 & Básica & Media & Superior & \\
\hline 1992 & $50,3 \%$ & $33,1 \%$ & $9,09 \%$ & $5 \%$ \\
\hline 2002 & $41,0 \%$ & $36,0 \%$ & $16,0 \%$ & $2,7 \%$ \\
\hline
\end{tabular}

Fuente: Instituto Nacional de Estadísticas (2006).

El aumento de cobertura posibilita el acceso a la educación y permite que, en un periodo de diez años, los índices de quienes solo tuvieron formación básica descendieran a un 4I\%, aumentando los porcentajes de personas que asistieron a la educación media e incrementando a I6\% el número de aquellos que tuvieron acceso a una educación superior (Instituto de Estadística INE, 2006). Finalmente, bajo el contexto de la Reforma Constitucional, en 2005 se aumenta la escolaridad obligatoria y gratuita a doce años.

Bajo el alero de un paradigma conductista, se llevaron a cabo mecanismos repetitivos que atribuían a la memoria la principal fuente que posibilita el aprendizaje. Las estrategias fueron homogéneas para todo el grupo, sin considerar características propias de la edad 
que tenían los alumnos o sus intereses y motivaciones. Este sistema brindaba rápidos resultados en relación a leer y escribir a una edad determinada, y las personas que eran evaluadas cumplían con requisitos básicos de lo que se ha considerado como "alfabetización".

En un análisis del INE (2006), con base en el censo de 2002, se menciona que, en Chile, la tasa de alfabetización de 95,7\%, lo que ubica al país entre los cinco mejores de América Latina, precisando que las expectativas de evaluación se relacionan con la denominada "alfabetización funcional", que apuntaba a las competencias bases de saber leer y escribir. Las investigaciones posteriormente incorporan elementos de reflexión crítica, participación en la comunidad y otras características relacionadas con la integridad de las personas, lo que nutre aquella perspectiva de educación funcional.

Hoy, a pesar de que las cifras de alfabetización de la población han aumentado, los estudios y resultados de distintas evaluaciones indican que no hay una relación entre saber leer y comprender lo leído. En el análisis de este nuevo contexto queda en evidencia que "más de un $80 \%$ de los chilenos entre 16 y 65 años no tiene el nivel de lectura mínimo para funcionar en el mundo de hoy" (Eyzaguirre, Foulon y Hinzpeter, 2000, p. 45). De este modo, se abren interrogantes sobre la importancia de la adquisición de herramientas que promuevan el aprendizaje de la lectura desde los primeros años, y preguntas en torno a cuál sería el mecanismo más adecuado para llevar a cabo el proceso de enseñanza. En consecuencia, se pone en la palestra investigativa cómo es posible potenciar sujetos reflexivos y críticos de sus propios aprendizajes en torno a la lectura, enriqueciendo el concepto de "alfabetización" en búsqueda de "competencias lectoras".

Por lo tanto, hoy los esfuerzos apuntan a visualizar el acto de leer como un campo que necesita ser potenciado, ya que se considera como una vía de acceso a la información y conocimiento del mundo. Ante esto Medina y Gajardo dicen que

[...] se requiere que las personas no sólo sean capaces de decodificar el significado de las palabras, sino que también se espera que sean capaces de manejar información de distinto tipo, leer comprensivamente los textos, comunicarse de 
forma eficaz, resolver problemas, desarrollar un pensamiento crítico, interpretar y evaluar los mensajes de los medios de comunicación, responder a un entorno en constante cambio, entre otras tareas. (Consejo Nacional de la Cultura y las Artes CNCA, 2015, p. I8)

Freire (2005) plantea que leer no se refiere al simple seguimiento de lo que escribe un autor o autora, sino que se pone en juego el relacionar la lectura o contenidos con la realidad o con los contextos, es decir, que se involucre todo el ser del sujeto lector -en sintonía con sus experiencias, su contexto personal y social- con aquello que se lee, pudiendo así tener la capacidad de "leer el mundo".

El concepto de "alfabetización" ha evolucionado acorde a las necesidades de un mundo globalizado. "Ahora la alfabetización se entiende como largo proceso que comprende todo el desarrollo humano, que cambia en las culturas y en la historia" (Braslavsky, 2003 , p. 3). Este proceso es entendido como una acción que requiere de acompañamiento, no solo por parte de un docente, sino que también por la familia, la sociedad y por políticas afines para un desarrollo acorde a los requerimientos.

\section{Recorrido histórico por la política de lectura en Chile}

¿Qué sucede a nivel nacional? ¿Cuáles son las propuestas y desafios que definen el acceso de los chilenos a la información impresa y digital? ¿A qué necesidades y/o carencias sociales responde la política de lectura?

Para contextualizar las posibles respuestas a estas interrogantes, es preciso remontarse a la cuenta pública del 2I de mayo de 20I4, en la que la presidenta de la República, Michelle Bachelet, anuncia la intención de modificar la política del libro y la lectura que se desarrollaba en Chile. "Crearemos el Plan Nacional de Lectura y una Política Nacional del Libro que haga de Chile un país de lectores y lectoras desde la primera infancia” (CNCA, 20I5, p. II). Estas últimas fueron las palabras, promesas y desafíos que proyectarían una política de carácter transversal que impactaría en la vida de cada uno 
de los chilenos, ofreciendo oportunidades para acceder a un mundo en el que las letras inspirarían, desde los primeros años de vida, el despertar de una sociedad más crítica, reflexiva y empoderada de un discurso creado y recreador de ideas.

Desde la década de los 90 los distintos gobiernos de turno, en su intento de generar una sociedad más democrática y con pensamiento crítico, fueron creando distintas propuestas que fortalecerían las habilidades de los ciudadanos frente a la lectura, esta última considerada como recurso, ventana de oportunidades y mecanismo para romper las barreras de la desigualdad.

Entre 2006 y 2010 se hizo efectiva la Política Nacional del Libro y la Lectura, que "sirvió de marco a distintos planes y programas de fomento lector en todas las regiones" (CNCA, 20I5, p. I5). Sin embargo y a pesar de las acciones realizadas, no logró la eficacia esperada debido a la falta de un sistema que permitiera evaluar el impacto y procesos correspondientes a éste. Es entonces, y asociado al cambio de gobierno, que esta ley entrará en un receso que durará por todo el nuevo mandato.

Hoy, dentro de las instituciones que colaboran en la implementación y seguimiento de esta política, están el Ministerio de Educación (MINEDUC), la Dirección de Bibliotecas, Archivos y Museos (DIBAM), la Corporación de Fomento de la Producción (CORFO), la Dirección de Asuntos Culturales (DIRAC), el Programa de Fomento a las Exportaciones Chilenas (ProChile), el Consejo de la Infancia y por supuesto que también los principales ejecutores y responsables, los educadores, que cada día están en el aula fortaleciendo el desarrollo del pensamiento divergente y analítico en sus estudiantes, mediante el recurso de la lectura.

\section{Factores influyentes en el desarrollo de la lectura}

Los factores influyentes en el desarrollo de la lectura trascienden a aquellos relacionados con aspectos físicos, emocionales, culturales y socioeconómicos. En países en los que se visualiza la exitosa implementación de planes lectores destaca el trabajo de los siguientes factores: 


\section{Formación del profesorado}

Contrariamente a la información obtenida de los planes y programas de países de la región iberoamericana, la formación del profesorado es un factor de mucho valor a la hora de implementar las mejoras en las diversas sociedades. El docente es visto como un elemento esencial en el aprendizaje de los niños. "La Comisión Europea (2008) destaca la calidad del personal docente como uno de los principales factores que determinan el éxito académico de los estudiantes, vital para lograr los objetivos de desarrollo económico y social de Europa” (Red de Información sobre la Educación Europea - Eurydice, 20II, p. 83).

Los gobiernos de los países con muy buenos resultados en sus lectores están pendientes de la formación que están recibiendo los profesores, atentos a las falencias para actuar rápidamente sobre ellas; se generan bastantes recursos para la capacitación y el profesorado es un gestor motivador importante para sus alumnos.

Otro factor predominante es la enseñanza de vocabulario. El profesor cumple un rol fundamental en la enseñanza del vocabulario; es de vital importancia mantener un lenguaje con nuevas palabras que los docentes utilicen diariamente para enriquecer el vocabulario de niños y niñas. Para contribuir en este factor, el profesor debe recibir una formación idónea que le permita aumentar progresivamente su vocabulario profesional. El docente debe utilizar términos técnicos que primeramente hayan sido trabajados, explicados y adquiridos, y debe continuar utilizándolos día a día, de forma integrada, para que los educandos no los olviden y puedan ayudar en la posterior comprensión lectora.

Se ha podido observar en las salas de clases de Chile el empobrecimiento del vocabulario a medida que pasan los años. Es también una problemática de orden común y, como plantean Biemiller y Slonim (200I, citado en Eurydice, 201 I),

la brecha en el vocabulario se amplía en los cursos superiores, es decir, los alumnos que comienzan su escolaridad con un vocabulario limitado, a medida que pasa el tiempo se distancian más de sus compañeros que comenzaron con un vocabulario más rico (p. 35). 
Otro aspecto vital en la formación profesional del docente es su conocimiento teórico y práctico de la conciencia fonológica. El profesor debe realizar un trabajo consciente, intencionado y profesional para llevar a cabo esta preponderante área de desarrollo para las bases de la lectura.

En muchos de los países mencionados en el estudio de Eurydice (20I I) esta área comienza a potenciarse en diferentes periodos según el país, pero con fuerte énfasis en la educación infantil. En algunos de los países con muy buenos resultados en el desarrollo de las competencias lectoras, el proceso se inicia en los centros de primaria. Es un trabajo que se toma en serio y en carácter de crucial.

Aproximadamente dos tercios de los países cuentan con documentos estratégicos que indican que el conocimiento de la fonología debe desarrollarse a lo largo de toda la primaria. En el resto de los países, la enseñanza de la fonología termina antes, normalmente hacia la mitad de la primaria o incluso antes, es decir entre los 7 y los I0 años. (Eurydice, 20I I, p. 57)

Por otro lado, los resultados de la investigación indican que el desarrollo de la conciencia fonológica y fonémica es necesario para la actualización de las habilidades tempranas, y cada vez se les está dando mayor importancia en los países europeos. Independiente de los periodos de tiempo que se dedica a esta área en cada país, se comienza habitualmente a trabajar en los primeros años de educación, siendo un factor no menor, ya que mejora y ayuda al desarrollo posterior de las competencias lectoras.

\section{Interés por la lectura}

En un sentido amplio, vivir en un ambiente en el que se valora la lectura es esencial para que un niño pueda llegar a ser un lector competente. Los modelos lectores que tenga un niño sin duda marcan diferencias en el placer que va a desarrollar por la lectura. Asimismo, el currículo finlandés establece que se debe mantener la actitud positiva de los alumnos hacia la lectura en todo momento; por esta razón, otro factor predominante dentro del fomento lector es la potenciación que se le da a la lectura fuera de la escuela, y un papel protagónico lo juegan 
las familias. Los padres que disfrutan leyendo y quieren compartir ese placer con sus hijos fomentan en ellos actitudes positivas hacia la lectura, promoviendo tiempos de ocio en torno al uso de libros y formando una completa comunidad de lectores.

Las actividades de lectura realizadas en el hogar, en familia, son fundamentales para el desarrollo de las habilidades lectoras de los niños, especialmente antes del comienzo de la escolaridad. La dedicación de las familias a la educación de sus hijos en general, y en el desarrollo de su capacidad lectora en particular, influyen en la motivación y en el rendimiento en lectura. (Eurydice, 20II, p. II3)

\section{Apoyo a estudiantes con dificultades}

Otro factor al que se le otorga mucha relevancia, tiempo y recursos, es al apoyo con programas específicos a los alumnos con dificultades lectoras desde el momento en que son detectadas. A modo de ejemplo, existe un modelo llamado "ADORE", proyecto europeo para enseñar a leer a adolescentes con dificultades.

En muchos países existen cursos dirigidos a profesores para ayudar a alumnos con dificultades y personal adicional para el apoyo de alumnos si es necesario. Como parte de la formación docente ya mencionada, se da un fuerte énfasis en la formación de especialistas de apoyo en el área de lectura, incluso los mismos docentes pueden tomar cursos para especializarse en dificultades de lectura.

En este sentido, toma mucha importancia la preparación de los docentes respecto de las necesidades y características de los estudiantes, para así tomar decisiones pedagógicas -en cuanto a metodologías y adaptaciones curriculares- que sean oportunas, pertinentes y significativas para el alumno que se apoya.

\section{Evaluación}

La evaluación es un área muy profundizada y dedicada, con énfasis en la evaluación formativa y a través de diversas estrategias que potencian la autocrítica, el autoaprendizaje y sobre todo el aprendizaje 
colaborativo. Los alumnos se ven enfrentados desde temprana edad a evaluar sus propios aprendizajes y a hacerse partícipes conscientes de su desarrollo. Es importante mencionar que en países como Finlandia se potencia la autoevaluación y la colaboración en el aprendizaje más que las evaluaciones estandarizadas, teniendo los profesores libertad para evaluar a sus alumnos.

Otro factor que se menciona ampliamente y que se considera de mucha importancia es la enseñanza de estrategias de comprensión lectora. Pero, por ser además uno de los referentes clave de la presente investigación, es un punto que se explicará de manera más profunda en el siguiente apartado.

\section{Estrategias de lectura}

Los educadores que entienden el proceso de la lectura utilizan métodos que la investigación ha probado como efectivos para ayudar a los niños a adquirir la comprensión del sistema de las lenguas alfabéticas. La mayoría de los niños tienen éxito al construir su propia comprensión, basándose en la metodología utilizada en las salas de clases.

Independientemente de la tendencia metodológica a la que se adscriba el educador, debe interiorizar un esquema didáctico que le permita facilitar al niño el acceso a la automatización del aprendizaje de la lectura. Este esquema está compuesto por estrategias que, en el marco de esta investigación, se relaciona con los procedimientos. Solé (2015) cita a Coll (I987) respecto de ello: "Un procedimiento -llamado también a menudo regla, técnica, método, destreza o habilidad- es un conjunto de acciones ordenadas y finalizadas, es decir, dirigidas a la consecución de una meta" (p. 58); sin embargo, es característico de las estrategias el hecho de que no detallan ni prescriben totalmente el curso de una acción. Su potencialidad reside precisamente en que son independientes de un ámbito particular y pueden generalizarse; su correcta aplicación requerirá de su contextualización para el problema de que se trate.

Un componente esencial de las estrategias es el hecho de que implican autodirección -la existencia de un objetivo y la conciencia de que ese objetivo existe- y autocontrol, es decir, la supervisión y evaluación del propio comportamiento en función 
de los objetivos que lo guían y la posibilidad de imprimirle modificaciones cuando sea necesario. (Solé, 2015, p. 59)

Bruner, citado por Aravena (2013), "define estrategia como un proceso de decisiones que involucra la búsqueda de claves para discriminar distintos aspectos que permitirán codificar el estímulo en categorías apropiadas” (p. 5I). Si bien los métodos que se utilizan son variados, depende de los educadores decidir cómo aplicarlos para conseguir un propósito específico. Lo importante es que los docentes deben tener un dominio de las estrategias a implementar para obtener buenos resultados. Según Swartz (2010), para que los educadores puedan dar esta clase de instrucción, necesitan saber qué conocimientos, habilidades y estrategias ya poseen los niños. Este entendimiento conjunto de teoría y práctica, es decir, con la observación de cada niño de manera continua y durante el proceso, es una aproximación efectiva a la lectura.

Solé (2015) destaca el papel de las estrategias lectoras como procedimientos de orden superior que implican lo cognitivo y metacognitivo, las cuales no pueden ser tratadas como recetas, sino más bien como la capacidad para representarse y analizar los problemas, y la flexibilidad para dar con soluciones. Por ende, en la enseñanza de estrategias lectoras hay que privilegiar la construcción y uso por parte de los niños de procedimientos generales que puedan ser transferidos a situaciones de lectura múltiples y variadas, de manera de asegurar su aprendizaje significativo.

\section{El papel de las estrategias en la lectura}

Enseñar estrategias de comprensión contribuye a dotar a los niños de recursos necesarios para aprender a aprender. Hacer lectores autónomos significa hacerlos capaces de aprender a partir de los textos. En este sentido, Palincsar y Brown (I984) se sitúan en una perspectiva cognitivista/constructivista, en la cual la comprensión de lo que se lee se debe a tres aspectos:

- La claridad y coherencia del contenido de los textos: que su estructura, vocabulario, sintaxis y cohesión interna sea familiar para los niños. 
- El conocimiento previo del lector frente al texto que lee, para que le atribuya significado a su contenido.

- Las estrategias que el lector utiliza para desarrollar la comprensión.

Por lo tanto, es necesario enseñar estrategias de comprensión para formar lectores autónomos, capaces de enfrentarse a diferentes textos; para ello deben ser capaces de interrogarse acerca de su propia comprensión, establecer relaciones entre lo que leen y lo que forma parte de sus conocimientos previos, cuestionar su conocimiento y modificarlo, estableciendo generalizaciones que permitan transferir lo aprendido a contextos distintos.

\section{¿Cómo podemos enseñar las estrategias de lectura?}

El objetivo de los educadores no debe ser formar niños que posean amplios repertorios de estrategias, sino que sepan utilizar las adecuadas para la comprensión del texto, las cuales se deben enseñar si los educadores no quieren que su potencialidad se diluya.

Palincsar y Brown (1984) proponen actividades cognitivas que deben ser desarrolladas mediante las estrategias:

I. Comprender los objetivos explícitos e implícitos de la lectura.

2. Activar los conocimientos previos respecto del contenido del texto.

3. Enfocar la atención a lo esencial del texto.

4. Evaluar el sentido y coherencia del texto.

5. Comprobar permanentemente si la comprensión sucede mediante la recapitulación y autointerrogación.

6. Elaborar y probar hipótesis, predicciones y conclusiones.

Desde la concepción constructivista de Coll (1990), las estrategias lectoras son entendidas como una ayuda que se proporciona al alumno para que pueda construir sus aprendizajes. La palabra "ayuda" cobra relevancia, ya que solo el niño puede realizar esa tarea y, a la vez, sin esa ayuda es muy difícil que el niño pueda dominar los contenidos de la enseñanza y lograr sus objetivos. 
Vamos a entender las situaciones de enseñanza y aprendizaje que se articulan alrededor de las estrategias de lectura como "procesos de construcción conjunta, en los que se establece una práctica guiada a través de la cual el profesor proporciona a los alumnos los "andamios" necesarios para que puedan dominar progresivamente dichas estrategias y utilizarlas una vez retiradas las ayudas iniciales" (Solé, 2015, p. 65).

Diversas propuestas teórico/prácticas se orientan en este sentido, como la de Swartz (2010) en su libro Cada niño un lector, quien se refiere a la liberación gradual de la responsabilidad, proceso que involucra la transferencia progresiva del trabajo que hace el educador directamente hacia aquel en el que los niños toman un papel más activo. AILEM UC (Aprendizaje Inicial de la Lectura, Escritura y Matemática), basado en el programa CELL (Aprendizaje Inicial de la Lectoescritura de California) y ExLL (Aprendizaje Extendido de la Lectoescritura) de Estados Unidos, cuyos principios orientadores son la transferencia progresiva de la responsabilidad de los aprendizajes, la autonomía y el trabajo colaborativo. Collins y Smith (1980, citados por Solé, 20I5) proponen una enseñanza en progresión a lo largo de tres fases: modelado, participación del alumno y lectura silenciosa. Baumann (I990, citado por Solé, 2015) sugiere la enseñanza directa de la comprensión lectora y divide en cinco etapas el método: introducción, ejemplo, enseñanza directa, aplicación dirigida por el profesor y práctica individual. Palincsar y Brown (1984) proponen un modelo de enseñanza recíproca, en el que el niño debe tomar un papel activo sobre la base de cuatro estrategias de comprensión de textos: formular predicciones, plantearse preguntas sobre el texto, clarificar dudas y resumirlo.

Es así que el dominio de las estrategias de comprensión lectora requiere progresivamente menor control por parte del educador $y$ mayor control por parte del alumno. "Se ha comprobado que una enseñanza que favorece el aprendizaje de un enfoque estratégico de la lectura, a través de un andamiaje adecuado, aumenta la confianza de los niños en su capacidad para leer textos nuevos" (Collins-Block, I992, en Villalón, 2008). 
Por su parte, Guthrie y Knowles (200I, citados por Villalón, 2008) señalan algunos riesgos que presenta la enseñanza de estrategias, los que es necesario considerar para lograr resultados efectivos en el aprendizaje autorregulado. Uno de ellos es que las estrategias deben ser enseñadas como instrumentos significativos para la comprensión. La enseñanza de estrategias desvinculadas de las necesidades de los niños puede resultar contraproducente más que motivador. Destacan también las necesidad de que los educadores brinden apoyo a los niños para ponerlas en práctica en situaciones nuevas, a través de actividades compartidas que constituyan una etapa intermedia en el proceso de adquisición de un desempeño independiente efectivo.

Como todas las propuestas, es necesario adecuar a cada contexto concreto con flexibilidad, es decir, que a partir de una visión global de lo que es el proceso de lectura, mediante la enseñanza se debe conseguir que los niños se conviertan en lectores activos y autónomos, que han aprendido de una forma significativa las estrategias responsables de una lectura eficaz y son capaces de utilizarlas independientemente en una variedad de contextos.

\section{Objetivos}

- Comparar estrategias lectoras aplicadas en el Plan Nacional de la Lectura con estrategias lectoras de planes lectores internacionales exitosos.

- Describir los factores que influyen en la implementación de un plan lector.

- Analizar la interrelación entre los factores influyentes en tanto facilitan u obstaculizan la implementación de un plan lector.

\section{Marco metodológico}

El tipo de investigación utilizado se enmarcó en el paradigma interpretativo, ya que nuestro fin era entender en profundidad la realidad lectora particular del Jardín Infantil Ricardo Levy. El diseño de la investigación fue descriptiva, detallándose las características que poseen los planes nacionales e internacionales de lectura, a partir de 
los cuales se describió cómo se implementa el plan lector en el nivel medio mayor y luego se analizaron los factores en tanto facilitan u obstaculizan su implementación. La metodología empleada es cualitativa, llevándose a cabo entrevistas y registros de observación.

Entre marzo y abril de 2017 se realizaron notas de campo (siete semanas), en los momentos de la jornada diaria en los que se realizaba lectura de textos hacia los niños y niñas, en el marco del Plan Nacional de la Lectura, en el nivel medio mayor de un jardín infantil de la comuna de Lampa.

Cada observación fue realizada en forma semanal, con una duración de 30 minutos, cuyo objetivo fue identificar cómo se implementa el plan lector en este nivel educativo, centrando los focos de observación en las interacciones adulto-niño, estrategias lectoras, ambiente físico, recursos, y otros que pudieran ir surgiendo en la práctica.

En paralelo a la aplicación de las notas de campo, se realizaron entrevistas a la educadora y técnicos de párvulos a cargo del nivel, como también a la directora del centro educativo. Su objetivo era conocer cómo se implementa el plan lector en el nivel medio mayor. Ambas técnicas e instrumentos de recolección de información tienen el mismo objetivo: comparar la práctica con el discurso de los actores involucrados.

La muestra se caracteriza por niños del nivel educativo Medio Mayor, ambos sexos, rango de edad 3 años.

Tabla 2. Información de la muestra

\begin{tabular}{|c|c|c|c|c|}
\hline CURSO & EDAD & NIÑAS & NIÑOS & TOTAL \\
\hline Medio Mayor & 3 años & I7 & I5 & 32 \\
\hline
\end{tabular}

Fuente: elaboración propia, 2019.

\section{Análisis de los resultados}

En el discurso de los actores se descubrió que, al paso del tiempo, se ha ido generando una despreocupación por el plan de lectura, así como también por la gestión de proyectos de fomento lector 
y la capacitación de mediadores. Si bien se detectó que, con anterioridad, hubo implementación de bibliotecas de aula y familiar, capacitaciones al personal y seguimiento constante de las prácticas, hoy la realidad que se evidencia es totalmente distinta. En la actualidad no existe una renovación de los recursos escritos que el jardín posee, lo que el equipo atribuye a una falta de interés por parte de la administración.

En relación a las notas de campo, los principales hallazgos se relacionan con la falta de estrategias lectoras, lo que evidencia que los agentes educativos no invitan a los niños a reflexionar sobre el texto leído y así identificar su nivel de comprensión. No favorecen que los niños hagan conexiones con su vida personal, sus experiencias y conocimientos previos. Donde se detectan mayores dificultades es en la utilización de estrategias después de la lectura: los agentes educativos solo realizan matutines de finalización y formulan escasas preguntas que inviten a los niños a recordar lo leído. En general, existe un desconocimiento por parte del equipo educativo del nivel respecto de la política o programa que sustenta el plan lector que implementan.

Se evidencia además la falta de participación por parte de las familias que, según el equipo educativo, no manifiestan interés por ser parte de los procesos educativos; sin embargo, éste tampoco presenta estrategias para realizar vínculos de estrecha alianza con los padres.

Respecto de la evaluación del plan lector, no cuentan con un sistema de seguimiento y monitoreo de éste, los resultados y el impacto del plan lector en los niños. Por otra parte, las modificaciones a lo largo del tiempo se realizan con base solo en las apreciaciones de los agentes educativos, por ende, la toma de decisiones se realiza en el nivel de las observaciones, pero falta el registro y el análisis de los procesos que se desarrollan en el plan de lectura.

\section{Conclusiones}

La información recolectada con la técnica "observación participante” en el nivel educativo investigado, permitió observar a los niños, niñas, educadoras y técnicos al interior del aula, momento 
en el que se levantaron diferentes hechos y situaciones durante la implementación del plan lector, aspectos que se relacionan con las categorías y subcategorías. Por lo tanto, fue necesario focalizar los temas en las categorías que tuvieran relación directa con los objetivos del trabajo de investigación, lo que permite concluir primeramente que existe un desconocimiento por parte del equipo educativo del nivel respecto de la política o programa que sustenta el plan lector que implementan. En el discurso de los actores, fuimos descubriendo que, al paso del tiempo, se ha ido generando una despreocupación por el proyecto, el cual en sus inicios fue acompañado por la "Fundación Había Una Vez". Si bien se detectó que durante la presencia de este organismo se lograron grandes avances, como la implementación de bibliotecas de aula y familiar, capacitaciones al personal y seguimiento constante de las prácticas, hoy la realidad es totalmente distinta.

En la actualidad el establecimiento cuenta con bibliotecas; sin embargo, no existe una renovación de los recursos escritos que posee, lo que el equipo atribuye a la falta de interés por parte de la administración. Por otro lado, la calidad y variedad de los recursos es insuficiente para lograr los objetivos descritos en el plan nacional, lo cual obstaculiza el desarrollo de prácticas que vayan en beneficio de sus usuarios (niños/as, familias y equipo).

En este mismo contexto, el equipo no es consciente de la utilización de otros recursos, como los descritos en el PNL, y reduce éstos solo al uso de textos impresos. Esta situación solo varía en el discurso entregado por la educadora del nivel, quien nombra los audicuentos y los títeres Kamishibay; sin embargo, este conocimiento no ha repercutido significativamente en el resto del equipo, ya que nadie más los menciona. Esta situación es una barrera que el mismo equipo levanta, al no manifestar interés por conocer, investigar y/o profundizar en la creación de un plan transformador, innovador y participativo.

En definitiva, la realidad del establecimiento, en relación a la generación de oportunidades referidas al acceso a la lectura temprana y todos los beneficios que ésta conlleva, se ven limitados a la mirada 
de un equipo que solo considera y ejecuta en relación a dos recursos: bibliotecas y material impreso.

Frente a los datos recogidos, se vuelve imprescindible remirar, reflexionar y evaluar el proceso de desarrollo de la implementación del PNL en este centro, y generar en sus actores un encantamiento y un sentido de responsabilidad social al respecto. Se sugiere entonces considerar la lectura oral tanto una actividad instructiva como también recreativa. Como se ha dicho, debe reunir el goce y el aprendizaje.

Al terminar los análisis de las entrevistas y las notas de campo, se detectaron diversas visiones y realidades en las prácticas entre los actores que participan en la institución educativa. Desde la visión de la directora, que hace referencia a una práctica consciente sobre el proceso del desarrollo de la comprensión lectora, que además siente y ve a su personal capacitado para trabajar de manera responsable en lo que concierne al Plan Nacional de la Lectura. Lamentablemente, las visiones llegan a ser tan diferentes unas de otras a tal punto de mencionar algunas de las técnicos: "en el equipo de trabajo no hay interés por la lectura y todo está mal, desde la educadora hacia abajo”. Declaraciones que hacen dudar de lo preparadas que están o se sienten tanto las educadoras como las asistentes de párvulos. Este ejemplo es una muestra referente de una institución en particular, pero lo más probable es que la misma situación puede estar ocurriendo en muchos jardines de nuestro país. Tomando en cuenta además que, según los discursos, el personal ha recibido capacitaciones en algún momento desde fundaciones de carácter privado para fortalecer el área de la lectura. Nos queda la inquietante duda, ¿qué ocurrirá en aquellas instituciones que sabemos nunca han recibido ningún tipo de capacitación relacionada con el desarrollo de la comprensión lectora? La respuesta se hace bastante evidente al revisar las fuentes estudiadas en la investigación, con los bajos porcentajes de adultos en Chile capaces de realizar lecturas de nivel avanzado.

Como se ha presentado durante el proceso de esta investigación, uno de los factores determinantes en el éxito durante el desarrollo de estrategias de comprensión lectora y buenos resultados es la oportuna 
capacitación al personal. En perjuicio de los niños y niñas de Chile, la capacitación aún continúa siendo un área lejos de las prioridades de gastos frecuentes de las instituciones y del Estado. Existe una deuda desde la formación profesional. Por lo que se logró evidenciar, existe poco conocimiento de adecuadas prácticas pedagógicas y estrategias lectoras que se puedan llevar a cabo desde la educación preescolar, lo que conlleva a malas prácticas educativas - sin ser mal intencionadas-, poco desarrollo de vocabulario desde el propio actor educativo y falta de comprensión lectora desde la que pretende enseñar comprensión.

Es de carácter urgente, a nuestros ojos, profundizar en la conciencia nacional sobre lo que modelamos frente a los niños a través de la lectura. Pero, ¿qué podemos hacer si el adulto mismo no se siente motivado? El interés por la lectura es otro factor preponderante mencionado en nuestra investigación. En los discursos se hace evidente un reduccionismo del plan lector a "libros en la sala", libros que podrían otorgar maravillosas experiencias de aprendizaje con las estrategias adecuadas y bien trabajadas, y lo que supera a todo lo demás... el encanto que demuestre el adulto por la lectura que tiene en sus manos, porque aquel encanto se podrá transformar en magia frente a los ojos de un niño que despierta en el desarrollo del proceso lector.

Cuando las instituciones y los propios maestros tomen conciencia de lo importante que es su propia actitud frente a la lectura y comprendan lo determinante que puede llegar a ser este modelo en la vida de un niño, en todos los aspectos, lograrán despertar el interés por la lectura en los niños y mantenerlo en el tiempo. Esta labor, claro, no es solo de las instituciones y profesores. Existe una gran tendencia de los padres y familias en nuestro país a desligarse de todos los procesos educativos de sus hijos y dejarlos en manos de los colegios como únicos responsables. Gran error, si bien existe un alto índice de vulnerabilidad en Chile respecto de recursos y escolaridad, los padres siempre van a ser el primer agente educativo, el primer agente encantador y el primer vinculo en el que nace todo desarrollo íntegro. Es evidente que cuando los padres muestran interés en las actividades, apoyan y son buenos modelos para sus hijos, los niños logran más avances. La sociedad actual de nuestro país está 
lejos de ser una sociedad modeladora de buenos lectores, mientras en países como Finlandia el paseo a la biblioteca es parte de la vida cotidiana. Pensemos quizás que nuestras familias también deben ser mayormente capacitadas en el desarrollo lector; por lo tanto, hacemos otro llamado de emergencia a los padres y a las familias de hoy a realizar "la lectura desde la cuna".

Otro de los elementos que nuestra investigación plantea como factores influyentes en desarrollo de un plan lector es la evaluación y, como se pudo constatar en los análisis, la evaluación carece hoy de profesionalismo, constancia y objetividad. Es más, en la muestra es casi inexistente. La evaluación debe ser un concepto que se renueve en beneficio de nuestros niños y transformarse en un proceso formador de aprendizajes y no como se plantea aún: una forma de terminar procesos. Los niños deben aprender a autoevaluarse y enfrentar la opinión de los demás como parte del desarrollo de personas reflexivas, críticas y de pensamiento divergente. Esta carencia de evaluación impide además detectar otro factor determinante en el desarrollo de las habilidades de comprensión en un niño: las dificultades en su aprendizaje. ¿Cómo podemos ayudar, apoyar y trabajar en estas falencias si no somos capaces de detectarlas de forma temprana? El apoyo oportuno es decidor para generar en niños con dificultades un aprendizaje que enriquezca sus habilidades, y este tipo de atención es prioridad en los países que generan muy buenos resultados en la comprensión lectora a través de sus programas. Otra deuda que debemos remediar en nuestro país.

Nuestra investigación ha querido presentar que no son solo los diversos modelos de planes lectores que se puedan aplicar, las estrategias que se puedan conocer, enriquecer, crear y/o aplicar en las diversas instituciones educativas, y los recursos utilizados; es la unión de un conjunto de factores mencionados lo que va a determinan el éxito de un plan lector. Es largo el camino que queda por recorrer y mucho trabajo en renovación de ideas, conceptos, actualización de conocimiento, capacitación, motivación personal y profesional el queda pendiente a nivel de país. 


\section{Referencias bibliográficas}

Aravena, M. (2013). Estrategias para Fomentar el Hábito Lector. (Tesis Doctoral). Universidad de Alcalá. Recuperado de: https://dialnet. unirioja.es/servlet/tesis?codigo $=$ I3238I

Braslavsky, B. (2008). Enseñar a entender lo que se lee: La alfabetización en la familia y en la escuela. Primera reimpresión. Buenos Aires, Argentina: Fondo de Cultura Económica.

Consejo Nacional de la Cultura y las Artes. (2015). Plan Nacional de la Lectura 2015-2020. I a edición. Chile.

Coll, C. (1990). Constructivismo y educación: la concepción constructivista de la enseñanza y del aprendizaje. Madrid: Editorial Alianza.

Eurydice. (20I I). La enseñanza de la lectura en Europa. Contextos, políticas y prácticas. Bruselas: Agencia ejecutiva en el ámbito educativo, audiovisual y cultural. (EACEA 99 Eurydice).

Eyzaguirre B., Le Foulon C. y Hinzpeter K. (2000). Los cbilenos no entendemos lo que leemos. Santiago de Chile: Centro de estudios públicos. Puntos de referencia.

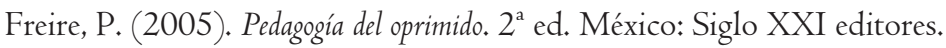

Instituto Nacional de Estadísticas. (2006). Día Internacional de la Alfabetización. Enfoque Estadístico. Recuperado de: http://www.ine.cl/filenews/ files/2006/septiembre/pdf/alfabetizacion.pdf

Solé, I. (2015). Estrategias de lectura. lector: Estrategias innovadoras para enseñar a leer y escribir. Barcelona, España: Editorial GRAÓ.

Swartz, S. (2010). Cada niño un lector: Estrategias innovadoras para enseñar a leer y escribir. Santiago de Chile: Ediciones Universidad Católica de Chile.

Villalón, M. (2008). Alfabetización inicial: claves de acceso a la lectura y escritura desde los primeros meses de vida. Santiago de Chile: Ediciones Universidad Católica de Chile. 